Photoshop 4 creates a new layer each time text is witten. For drawing an arrow, create a new layer first.

2) For the layer of interest, deselect the "preserve transparency" feature in the layers palette.

3) Make white your foreground color.

4) Holding down the "command" button ("ctrl" an a Wintel), click on the layer of interest in the layers palette. This selects the non-transparent areas of the layer (e.g., your text or feature).

5) Under the Edit menu, select "stroke". This will put a line of the foreground color, at a selected width, on the inside, outside, or center of the dashed "select" line in the image. A five pixel width for a 300 dpi image and "outside" are typical. 6) Select OK; this should give the text or feature a white border. This can be seen more easily (without deselecting the feature) by using the "Hide Edges" command (command-H for Macintosh). This removes the dashed 'select" line (though the feature is still selected), and allows a clean look at the work for proofing.

John Vetrano will welcome questions on this procedure and may be contacted at email address: js_vetrano@pnl.gov

John Vetrano, Pacific Northwest Laboratory

\section{Healing Cracks in Glycolmethacrylate Embedded Sections:}

Soak the slide with attached sections in a coplin jar with acetone for 10 minutes or so (I have gone up to 30 or 40 minutes), rinse with water, and then apply your stain while the sections are still wet.

Generally the cracks seem to disappear, or are at least minimized. and it no longer interferes with the microstructure of whatever l'm looking at.

I typically use this for sections of between 2 and $5 \mu \mathrm{m}$ sections for light microscopy.

I haven't figured out where the cracks come from. Initially I thought It was age, (old sections) but have also had problems with freshly sectioned material. (including freshly embedded and polymerized)

\section{S. Shea Miller, Agriculture \& Agri-Food Canada}

\section{Archival Storage of Polaroid Films}

The materials listed below contain a minimum of potentially harmful substances. Therefore, they are regarded as being among the more suitable for the storage of photographs.

+ Storage boxes made of acid-free paper or card - Cellulose acetate

+ Polyethylene

+ Polyester sheeting and sleevesas

+ Aluminum

+ Stainless Steel

+ Materials coated with baked enamel

+ Glass

+ Porcelain

+ Acrylic plastics

The following products should be avoided:

- Paper with a high content of sulfur compounds (such as some black album papers)

+ Brown kraft paper (contains lignin and metallic sulfates)

+ Unstable plastics (such as polyvinyl chloride and polystyrene)

+ Ordinary cardboard

+ Some uncoated and unaged wood, especially those containing glue (plywood or pressboard)

+ Wood recently varnished or painted

+ Glassine envelopes

+ Inks from pens or rubber stamps

+ Rubber bands(may contain sulfur)

+ Insecticides and fungicides

Philip J. Cravotta, Polaroid Technical Assistance

\section{Mounting Small Specimens for Making an Animated Sequence in the SEM:}

I'm doing an animated sequence from the SEM, in stereo, on a very small spider (a couple of thousand frames). The mounting technique is critical for doing this.

\section{Tip \#1: BSE detection and Carbon}

I'm shooting with a Robinson BSE detector. It gives a more "real" illumination than SE and has the benefit of not "seeing" carbon. The specimen is normally mounted on top of a minuten (entomological) pin which is clamped in a $12 \mathrm{~mm}$ "vice-stub" and tilted at 90 degrees in the $\mathrm{SEM}$. On my Cambridge $\mathrm{S} 120$, the top of the stage mechanism is now out of view but you still see the bottom plate below. I've covered that with a piece of aluminum foil which has been painted with thinned-out carbon dag, making it a perfectly black, "studio-shot" background when using BSE.

Tip \#2: No unsightly props

Further, instead of mounting the specimen on the usual minuten pin, I've fixed it to the end of the lead from a clutch pencil (draughtsman's pencil), so the pin is also virtually invisible under BSE.

Doesn't the pencil lead get sputter-coated too? I've taken a mediumbore tip from a syringe, held it in an alligator clip, and fed the pencil lead down into it leaving just the specimen exposed for coating.

Tip \#3: Accuracy in mounting

Mounting very small specimens on a pin-tip is difficult at the best of times. As the animation sequence is to be in stereo, the specimen must be at exactly the right angles to the pin and we don't want to see any excessive blobs of carbon dag. You'll need a small bench vice, micrometer screw gauge and double sided tape (DST). Lay your stereomicroscope down on its front with the head reversed so that the eyepieces point up and the light path is (roughly) horizontal. Clamp the side of the screw gauge into the side of the vice and place it in front of the stereoscope. Secure your specimen to a foam block with crossed minuten pins and secure the block in position to the opposing pole of the screw gauge with DST. Fix the mounting pin to the mobile shaft of the screw gauge with DST. Watching it all through the stereoscope, do a dummy run to ensure that everything lines up. Screw the pin up, apply a minute amount of dag to the pin, just enough to form a meniscus on the squared-off end of the pin (rather than a blob) and screw it down to the specimen. Leave to dry.

\section{Tip \#4: Centre of rotation}

Because a sequence may include rotating the animal 360 degrees, the pin has to be at the centre of rotation of the stage. However, in my 12 $\mathrm{mm}$ "vice-stub", the vice is offset. Instead, I'm using a pop-rivet. Push the nail out and use the rivet and its collar; the rivet has the same diameter as the shaft on a normal $12 \mathrm{~mm}$ grooved stub. Put the pin down the bore and dag it into a central position.

I hope you find these tips helpful and it you're in $\mathrm{Oz}$ around September, drop into the Australian Museum, Sydney, to see the stereo animation sequence of the spider (unfortunately, I'll be gone by then).

\section{Geoff Avern, Australian Museum, Sydney}

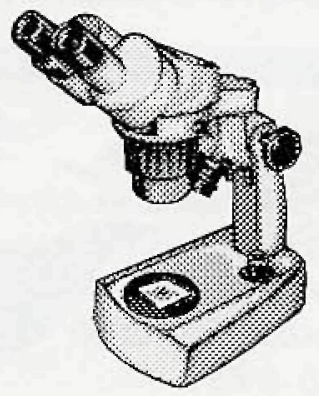

